\title{
ERRATA, VOLUME 13
}

R. D. Carmichael, On Euler's $\phi$-function, pp. 241-243.

The author has been informed by Professor E. A. Hedburg that the second theorem may be stated as follows:

The number of solutions of $\phi(x)=2^{n}$ is $n+2$ when $n \leqq 31$, and exactly 32 when $31<n<1024$.

This corrects the result as originally stated by having 32 in place of 33 (as formerly given) and extends the result by having 1024 as the upper bound for $n$ instead of 256 as in the initial paper. In making the extension, Professor Hedburg uses the now known facts that the numbers $2^{28}+1$ and $2^{29}+1$ are composite.

\section{ERRATA, VOLUME 53}

S. M. Shah, On real continuous solutions of algebraic difference equations.

p. 550, line 17. For " $\left\{f\left(x_{0}\right)\right\} \boldsymbol{B}_{p}$ " read “ $\left\{f\left(x_{0}\right)\right\} \boldsymbol{B}^{p}$."

p. 558, last line. For "(30)" read "(29)."

Index of Volume 53.

p. 1198, Hall, M. For "(1111)" read "(1110)."

p. 1199, Stoll, R. R. For “(1113)" read "(1112)."

\section{ERRATA, VOLUME 54}

S. P. Avann, Ternary distributive semi-lattices. Abstract 54-1-86.

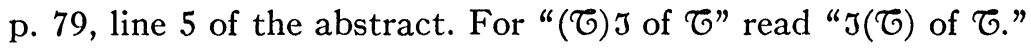

Bjarni Jónsson and Alfred Tarski, Representation problems for relation algebras. Abstract 54-1-89.

p. 80 , line 3 of abstract. For " $a \mid \overline{a \mid b}$ " read " $a-\mid \overline{a \mid b . "}$

p. 80, line 6 of abstract. For " $a \in U$ " read " $(a, a) \in U$."

p. 80, line 11 of abstract. For "a" read " $a$ ", twice.

Albert Newhouse, On finite extending groups.

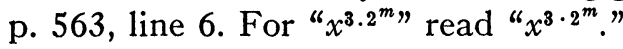

P. M. Morse, Mathematical problems in operations research.

p. 603 , last line. For "mean" read "men."

J. S. Frame, Group decomposition by double coset matrices.

p. 748, display (4.15). For " $\left(Q_{j}^{t \prime}\right)$ " read " $\left(Q_{j}^{t}\right)^{\prime}$."

Notes. p. 870, line 11. For "Rundberg" read "Rudberg." 